\title{
OUTGOING EDITOR'S NOTE
}

This is Esther Kim Lee's first issue as Editor of Theatre Survey, and she is being joined now by her Associate Editor, Harvey Young. Esther and Harvey are both award-winning scholars and have extensive experience as editors, and I am extremely honored by this opportunity to leave the journal in such excellent hands. Theatre Survey is starting its new life as a tri-annual publication, and the challenges ahead are both exciting and daring. As our last conference in Nashville showed, we are able to weather natural and economic storms together, and ASTR is a welcoming home to different generations, voices, and opinions. We are moving towards a magnificent future together. 